\title{
A first start for lung transplantation?
}

\author{
Jonathan D'Cunha, MD, PhD
}

\author{
From the Division of Lung Transplantation and Lung Failure, Department of Cardiothoracic Surgery, University \\ of Pittsburgh Medical Center; Pittsburgh, Pa \\ Disclosures: Author has nothing to disclose with regard to commercial support. \\ Received for publication Aug 26, 2018; accepted for publication Aug 27, 2018. \\ Address for reprints: Jonathan D'Cunha, MD, PhD, Department of Cardiothoracic Surgery, University of Pitts- \\ burgh Medical Center, UPMC Presbyterian Suite C-900, 200 Lothrop St, Pittsburgh, PA 15213 (E-mail: \\ dcunhaj@upmc.edu). \\ J Thorac Cardiovasc Surg 2018;156:2353-4 \\ $0022-5223 / \$ 36.00$ \\ Copyright (c) 2018 by The American Association for Thoracic Surgery \\ https://doi.org/10.1016/j.jtcvs.2018.08.065
}

They told me there was not enough blood available. "YOU CANNOT BE SERIOUS,' I said in a not-so-satisfied tone. A donor was available for a 20-year-old inpatient with pulmonary fibrosis and short telomere syndrome who had associated dyskeratosis congenita and myelodysplastic syndrome. This would be reoperative chest surgery because of a previous right middle lobectomy. The patient was listed for lung and bone marrow transplants through a clinical protocol in which we do the lung transplant first and 3 months later the bone marrow transplant occurs. With his bone marrow dysfunction a known substantial comorbidity, platelet counts were in the range of 10,000 cells $/ \mathrm{mm}^{3}$, and his white blood cell count was supported with filgrastim. The patient had waited for a long time for this bone marrow match, and so we could not pass this opportunity up as he was receiving $25 \mathrm{~L}$ oxygen via nasal cannula and progressively declining. He had been an inpatient for months. We needed time to coordinate. The donor operating room was ready, and they tried to accommodate as much as possible, but we were not ready in Pittsburgh. Our recipient also needed a bone marrow match, and this was literally his one chance as he had entertained many offers to date that had no good HLA matches in donors with good lungs. We went out to get the lungs, harvested them, and brought them back to Pittsburgh. They were placed on ex vivo lung perfusion (EVLP) at a point at which we were ready to move forward with the recipient and his blood products were available. We also now had our daytime team for what was anticipated to be a significantly complex transplant with a first start. We were able to request one of our best anesthesiologists. The blood bank director was physically ready to help in the operating room.

Lung transplantation is a lifesaving procedure for those with end-stage lung disease. The field has enjoyed tremendous growth and excitement in the past several years. The implementation of EVLP has opened the door to a new era in lung transplantation. In their editorial in this issue of the Journal, Cypel and colleagues ${ }^{1}$ introduce the concept of the

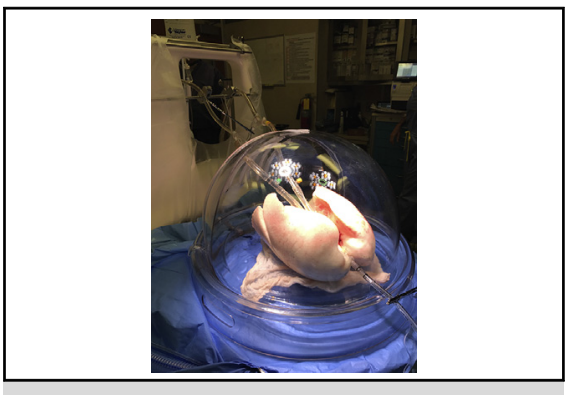

Lungs on the circuit in the light of day.

Central Message

The era of ex vivo lung perfusion for transplantation has arrived and opens the door to many strategies in donor organ management. A semielective reality for the operation is upon us.

See Article page 2350.

"semielective" lung transplant facilitated by EVLP. This is a timely article highlighting important preclinical and clinical developments in how we manage the practice of lung transplantation from day to day. In addition to highlighting important areas of investigation into ischemic times, Cypel and colleagues ${ }^{1}$ define nomenclature of the various phases of the transplant such that all can use universal definitions when comparing data and outcomes among centers. EVLP can help prolong donor lung preservation times without adverse effects to recipients, and the doors have opened to considering additional therapies to improve quality and function. Originally developed with the vision of increasing the number of organs available for transplant, the evolution of its potential has brought forth numerous downstream enlightenments to the field. Managing logistics from donor evaluation to recipient transplant is just one of these potentially very important multifarious areas of impact.

Our patient got his transplant successfully and recently received his bone marrow transplant. A first start for his case with the right team and resources was absolutely critical to success. As Cypel and colleagues ${ }^{1}$ point out, we are clearly entering an era in which EVLP is a tool to make semielective lung transplantation a reality for patients. When applied thoughtfully, the downstream advantages suggest more lungs for more recipients while potentially affording clinical advantages for lung transplant programs. As the cost and expertise of such technology are 
investigated deeper, it is further believed that centralized EVLP centers will help drive all lung transplant centers to serve their patients in a positive way.

\section{Reference}

1. Cypel M, Yeung JC, Keshavjee S. Introducing the concept of semielective lung transplantation through the use of ex vivo lung perfusion. J Thorac Cardiovasc Surg. 2018;156:2350-2. 